\title{
Música y emocionalidad: efectos de la música sobre el estado de ánimo y la memoria verbal
}

Music and emotionality: effects of music on mood and verbal memory

Julieta Moltrasio', Florencia Dominguez², Verónica Detlefsen³, Wanda Rubinstein ${ }^{4,2,1}$

\section{Resumen}

Los estímulos emocionales son mejor recordados que los estímulos neutros. La música, como estímulo emocional, modula la memoria de estímulos de otra modalidad; puede cambiar el estado de ánimo y generar un mejor recuerdo de material congruente con él (hipótesis de congruencia). El objetivo del presente trabajo es estudiar el efecto de la música activante y relajante sobre la memoria verbal emocional en adultos jóvenes, y analizar el efecto de la congruencia del estado de ánimo sobre el tipo de material recordado. Se les administró una lista de palabras, positivas, negativas y neutras, a 33 adultos. Se utilizó música positiva activante y relajante, o ruido blanco, como tratamiento post-aprendizaje. Se midió el estado de ánimo. Se les tomó una tarea de recuerdo inmediato. Los resultados indicaron un mejor recuerdo de palabras totales y negativas en el recuerdo libre en sujetos expuestos a música activante. La música activante y relajante generó disminución de la ansiedad, en tanto que la condición control generó un aumento de la hostilidad. Estos resultados apoyan la idea de que la música puede ser utilizada como tratamiento para modular recuerdos verbales, aunque no se halló efecto de congruencia con el estado de ánimo.

Palabras clave: Música - Memoria verbal - Congruencia - Estado de ánimo - Emociones.

\begin{abstract}
Abtract
Emotional stimuli are better remembered than neutral stimuli. Music, as an emotional stimulus, modulates memory; it can change the mood and improves memory of material congruent with it (congruence hypothesis).

The aim of this work is to study the effect of activating and relaxing music on emotional verbal memory in young adults, and to analyze the effect of mood congruence on the type of material remembered.

33 adults listened to a list of positive, negative and neutral words. Activating and relaxing positive music, or white noise, was used as a post-learning treatment. Mood was measured. We performed an immediate recall task.

The results indicated better recall of total and negative words in free recall in subjects exposed to activating music. The activating and relaxing music generated a decrease in anxiety, while the control condition generated an increase in hostility. These results support the idea that music can be used as a treatment to modulate verbal memories, although no effect of congruence with mood was found.
\end{abstract}

Keywords: Music - Verbal memory - Congruence - Mood - Emotions.

\footnotetext{
RECIBIDO 30/9/202 I - ACEPTADO 3/I2/202 I

1. Universidad de Buenos Aires, Facultad de Psicología, Instituto de Investigaciones. Buenos Aires, Argentina.

${ }^{2}$ Centro de Investigaciones en Neurociencias y Neuropsicología, Universidad de Palermo. Buenos Aires, Argentina.

3. Universidad de Buenos Aires, Facultad de Psicología. Buenos Aires, Argentina.

4.HIGA “Eva Perón”, CONICET, Laboratorio de Deterioro Cognitivo. San Martín, Buenos Aires, Argentina.
}

\section{Autora de referencia:}

Julieta Moltrasio

julietamoltrasio@gmail.com

Lugar de realización del estudio: Instituto de Investigaciones, Facultad de Psicología, Universidad de Buenos Aires. 


\section{Introducción}

La emocionalidad de un estímulo, según el modelo dimensional, puede definirse de acuerdo al nivel de activación o arousal, que define al estímulo en términos de cuánta activación o relajación genere en el sujeto; y la valencia, que determina cuán agradable (positivo) o desagradable (negativo) es (Bradley \& Lang, 2000; Kensinger \& Corkin, 2003). Los estímulos emocionales generan una activación mayor que los estímulos neutros, y modulan los recuerdos (Cahill \& McGaugh, 1995; Justel et al., 2013). Es decir, aquellos estímulos que son calificados como activantes, son mejor recordados que los estímulos neutros (Bradley et al., 1992; Cahill et al., 1996).

Diversos estudios utilizaron música, como estímulo emocional (Lundqvist et al., 2009; Kreutz et al., 2008), para modular el recuerdo de estímulos verbales y visuales. La música activante se caracteriza por presentar un tempo rápido, cambios inesperados en la melodía, ritmo e intensidad, y uso de instrumentos de timbres estridentes, mientras que la música relajante se caracteriza por un tempo lento, ritmo repetitivo, cambios esperables en la melodía y uso de instrumentos con timbres cálidos (Chanda \& Levitin, 2013; Knight \& Rickard, 2001). En cuanto a la valencia, se consideran positivos aquellos extractos compuestos en modo mayor (Vieillard et al., 2008).

Algunos estudios utilizaron música activante (que genera altos niveles de arousal) en la etapa de consolidación, como tratamiento post-aprendizaje, para mejorar el recuerdo de estímulos visuales (Justel et al., 2013; Justel et al., 2016). También se utilizó la música para modular el recuerdo de estímulos verbales neutros y emocionales (Judde \& Rickard, 2010; Justel et al., 2016; Moltrasio et al., 2020; Rickard et al., 2012). Los resultados sugieren que la música activante mejora el recuerdo de estímulos verbales emocionales, en un recuerdo inmediato (Moltrasio et al., 2020), recuerdo diferido con un intervalo de 30' ( Justel et al., 2016) y con un intervalo de una semana (Moltrasio et al., 2020). La música relajante impide que se produzca el efecto de modulación del material emocional verbal (Rickard et al., 2012).

Otros estudios sugieren que la música modifica el estado de ánimo del oyente (Bruner, 1990; Jallais \& Gilet, 2010; Martin, 1990; Oetken et al., 2017). La música relajante generaría una disminución de la ansiedad (Knight \& Rickard, 2001). Además, el material recordado en cierto estado de ánimo, inducido musicalmente, será congruente con dicho estado de ánimo (Västfjäll, 2001).
Bower (1981) planteó que cada estado de ánimo estaría representado en un nodo perteneciente a una red de cogniciones y recuerdos: cuando el nodo es activado, provoca la evocación de los contenidos mnésicos asociados con el estado de ánimo en cuestión (Martin, 1990). Los estados de ánimo tienden a incrementar la consolidación y la accesibilidad del contenido congruente con él: el material positivo será mejor codificado y posteriormente recordado en estado de ánimo alegre, mientras que el contenido negativo lo será para los estados de ánimo tristes (Mayer et al., 1995). Diversas investigaciones comprobaron la hipótesis de la congruencia del estado de ánimo, utilizando música para inducir el estado de ánimo antes de presentar el estímulo a recordar (Clark \& Teasdale, 1985; Mayer et al., 1990; Tesoriero \& Rickard, 2012). Otras investigaciones no hallaron evidencia a favor de esta teoría en todos los casos (Knight \& Rickard, 2001; Nasby, 1994).

De acuerdo a la hipótesis de congruencia, la música activante de valencia positiva, al generar un estado de ánimo alegre (Västfäll, 2001; Tesoriero \& Rickard, 2012), generará un posterior recuerdo de material positivo (Tesoriero \& Rickard, 2012). Sin embargo, en un estudio llevado a cabo por Sheldon, Williams, Harrington, \& Otto (Sheldon et al., 2020), utilizaron música para favorecer la evocación de recuerdos autobiográficos, y hallaron que solo el nivel de arousal, pero no la valencia, predijo cuán detallados fueron los recuerdos evocados. Además, en un estudio reciente, se halló mejor recuerdo de material negativo con un estímulo musical agradable (positivo) presentado en la etapa de consolidación (Moltrasio et al., 2020), aunque no se midió el efecto de la música sobre el estado de ánimo. No existen estudios que hayan utilizado la música como tratamiento post-aprendizaje y hayan tomado medidas de estado de ánimo para comprobar el efecto de la música sobre este.

El objetivo del presente trabajo es estudiar el efecto de la música activante y relajante sobre la memoria verbal emocional en adultos, y analizar el efecto de la congruencia del estado de ánimo sobre el tipo de material recordado. Partimos de la hipótesis de que se obtendrá un mejor recuerdo con la música activante que con la música relajante y el ruido blanco, y que, además, se verá beneficiado el recuerdo de material positivo, congruente con el estímulo musical.

\section{Metodología Participantes}

Treinta y tres adultos (19 mujeres, 14 hombres) jóvenes entre 23 y 33 años $(M=27,3 \mathrm{DE}=3,2)$, con más de 
12 años de escolaridad $(\mathrm{M}=16,4 \mathrm{DE}=1,8)$, residentes de la provincia de Buenos Aires.

Se tomaron los siguientes criterios de exclusión: Consumo de sustancias; depresión, medida a través del Inventario de Depresión de Beck (BDI-II) (Beck et al., 2006); historia de enfermedades psiquiátricas y neurológicas; deterioro cognitivo, evaluado mediante el Mini-Mental State Examination (MMSE) (Folstein, \& McHugh, 1975; Butman et al., 2001); y sujetos con 5 o más años de formación musical.

\section{Instrumentos}

\section{Cuestionarios demográficos}

Se utilizó un cuestionario de datos personales: edad, escolaridad, consumo de sustancias psicoactivas (café, mate, té, alcohol, cannabis, anfetaminas) y psicofármacos, las horas previas a la evaluación, antecedentes psiquiátricos, enfermedades relevantes, años de educación musical. Además, completaron un Cuestionario de preferencias musicales, que indagaba acerca de géneros musicales predilectos y contacto actual con la música (escuchar, bailar, etc.) (Mercadal-Brotons, \& Augé, 2008).

\section{Estado de ánimo}

Para evaluar el Estado de ánimo se utilizó la Escala de Valoración del Estado de ánimo (EVEA) (Sanz Fernández, 2001), que contiene 16 ítems, pertenecientes a 4 subescalas: Ansiedad, Hostilidad, Depresión y Alegría. Los sujetos deben puntuar cada ítem (de 1 a 10), de acuerdo a cómo se sienten en ese preciso momento. Esta escala ha sido utilizada en estudios que aplican estímulos musicales para inducir estados de ánimo (Sanz Fernández, 2001; Castro, 2018; Romero, 2018).

\section{Memoria verbal emocional}

Se utilizó una lista de 40 palabras, tomadas de la versión en español del Affective Norms for English Norms (ANEW) (Bradley \& Lang, 1999; Redondo et al., 2007), balanceadas por frecuencia y familiaridad. Se seleccionaron 20 palabras neutras (Media de arousal = 4,9), 10 palabras negativas (Media de arousal $=6,24$ ) y 10 positivas (Media de arousal $=6,27$ ). Estas mismas palabras habían sido utilizadas en un estudio previo (Moltrasio et al., 2020).

\section{Grilla de calificación de las palabras delANEW}

Para la calificación de los estímulos verbales se utilizó una grilla de emocionalidad, de acuerdo al nivel de arousal. El nivel de arousal oscilaba entre 1 y 5 , siendo $1=$ nada de activación, $2=$ poca activación, $3=$ moderada activación, $4=$ mucha activación y $5=$ muchísima activación.

\section{Tratamiento}

Los estímulos utilizados como tratamiento post-aprendizaje fueron: música activante agradable (positiva), música relajante agradable y ruido blanco. Éste último ha sido utilizado como condición control en estudios de modulación de la memoria (Justel \& Rubinstein, 2013; Rickard et al., 2012). Como estímulo activante se utilizó la Sinfonía de Haydn Joseph No. 70, Re Mayor, utilizada previamente para inducir emociones positivas (Kreutz et al., 2008), y como tratamiento post-aprendizaje en estudios de modulación de la memoria (Justel \& Rubinstein, 2013; Justel et al., 2016). Para la música relajante, el canon en Re mayor de Pachelbel, utilizada previamente para reducción de la ansiedad (Knight, \& Rickard, 2001), y como tratamiento musical en estudios de modulación de la memoria (Justel et al., 2016). Ambos estímulos musicales buscan inducir un estado de ánimo positivo (alegría), y, en el caso del estímulo relajante, reducción de la ansiedad.

\section{Procedimiento}

Utilizamos un procedimiento similar a un estudio anterior (Moltrasio et al., 2020), aunque sin utilizar un recuerdo diferido, y tomando una medida de estado de ánimo antes de la presentación de las palabras, y una después de la música.

Primero se les solicitó a los sujetos que firmaran un consentimiento informado. Todos los participantes firmaron un consentimiento informado a los fines de esta investigación. Se administró al total de los participantes las escalas y tests de exclusión: BDI-II y MMSE, seguido de cuestionarios de datos personales y preferencias musicales, y la Escala de Valoración de Estado de Ánimo (EVEA). A continuación, se leyó una lista de palabras en voz alta, en un orden aleatorio. Luego de escuchar cada palabra, cada participante debía puntuar de acuerdo al nivel de arousal (grilla de calificación de las palabras).

Inmediatamente después de escuchar la lista de palabras, fueron expuestos a 3' de música o ruido blanco, de acuerdo al tratamiento correspondiente: 11 sujetos fueron expuestos a ruido blanco, 11 a música activante y 11 a música relajante. Fueron asignados aleatoriamente a cada condición. Se utilizaron auriculares para presentar los estímulos, y fue solicitado que prestaran atención únicamente a la pieza musical o ruido blanco. Luego, completaron nuevamente la EVEA. Después, debían evocar libremente la mayor cantidad de palabras de la lista. 


\section{Análisis estadístico}

Se utilizó análisis de varianza (ANOVA) para las comparaciones intra-grupo. Para analizar los datos de edad y escolaridad se realizó un ANOVA de una vía, siendo Tratamiento el factor intersujeto. Para evaluar la variable de arousal se empleó un ANOVA de medidas repetidas (MR), siendo Tratamiento el factor intersujeto y la media de activación para cada tipo de palabra la MR.

Para analizar el efecto de la música sobre el estado de ánimo, se realizó un ANOVA MR, siendo las medidas pre y post (EVEA pre y EVEA post) el factor intrasujeto, y el Tratamiento (activante, relajante o ruido blanco), el factor intersujeto. Se realizó este análisis por cada sub-escala del EVEA (alegría, ansiedad, hostilidad, depresión) por separado.

Se ponderaron los puntajes brutos de recuerdo inmediato de cada sujeto, ya que las palabras neutras totales eran el doble (20 palabras) que las palabras negativas y positivas totales (10, respectivamente). Se procedió dividiendo la cantidad recordada sobre el total de palabras (10 para las negativas y 10 para las positivas, y 20 para las neutras). Luego se realizó un ANOVA MR, siendo Tratamiento el factor intersujeto y la ponderación de palabras (para cada tipo de palabra) recordadas la MR. El p valor se fijó en 0,05 y se calculó el Eta cuadrado parcial $\left(\eta^{2} \mathrm{p}\right)$ para estimar el tamaño del efecto.

El programa utilizado para el análisis estadístico fue el Statistical Package for the Social Sciences (IBM SPSS Statistics 25).

\section{Resultados}

\section{Datos sociodemográficos}

No se hallaron diferencias significativas entre grupos en cuanto a edad, $\mathrm{F}(2,32)=0,402, \mathrm{p}=0,673$; ni escolaridad, $\mathrm{F}(2,32)=0,249, \mathrm{p}=0,781$.

En relación al arousal, se halló un efecto significativo del factor Palabra F $(2,29)=100,811, \mathrm{p}<0,0001, \eta^{2} \mathrm{p}$ $=0,771$. El análisis post-hoc determinó que las palabras positivas $(M=4,1, D E=0,4)$ y negativas $(M=4, D E=$
$0,8)$ fueron calificadas por los sujetos como más activantes que las palabras neutras $(M=2,4, D E=0,4)$, pero no difirieron entre sí.

\section{Estado de ánimo}

El ANOVA MR aplicado a Depresión y Alegría, no halló ningún efecto $(\mathrm{F}(2,30)=0,964, \mathrm{p}>0,334, \mathrm{~F}(2,30)=$ $0,163, p>0,689)$. Se halló un efecto significativo de la interacción pre-post $\mathrm{x}$ Tratamiento $\mathrm{F}(2,30)=6,864$, $\mathrm{p}$ $<0,005, \eta^{2} \mathrm{p}=0,337$ en el ANOVA MR aplicado a Ansiedad. El análisis post-hoc indicó una disminución significativa de la ansiedad en el grupo expuesto a música relajante. La figura 1 muestra una disminución de la ansiedad en el grupo expuesto a música relajante y un aumento en el grupo expuesto a Ruido Blanco, aunque ésta no fue significativa.

\section{Figura I.}

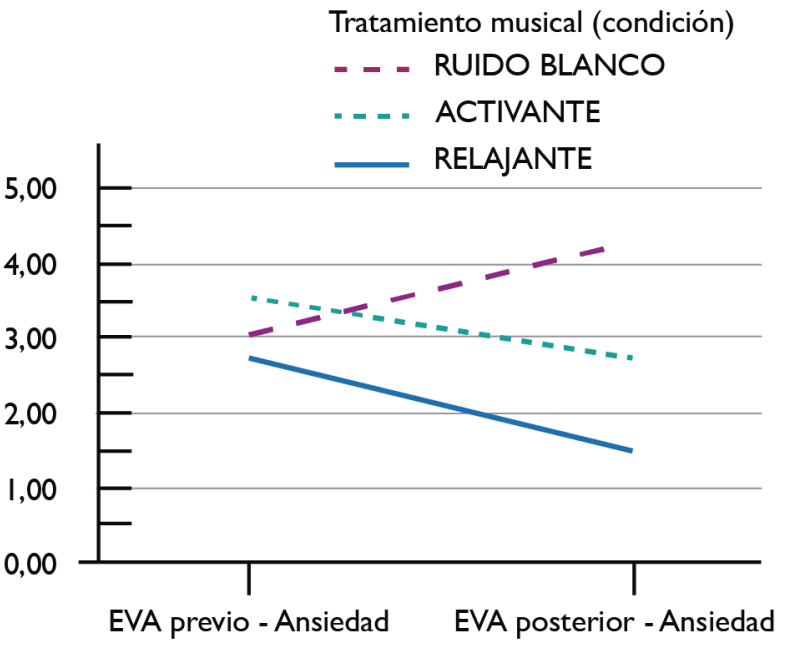

También se halló un efecto significativo en la subescala de Hostilidad, a nivel intragrupo (pre-post) $\mathrm{F}(2,30)=4,334 \mathrm{p}=0,046, \eta^{2} \mathrm{p}=0,126$, siendo mayor la media de Hostilidad post-tratamiento en todos los grupos. También resultó significativa la interacción pre-post $\mathrm{x}$ Tratamiento $\mathrm{F}(2,30)=10,717, \mathrm{p}<0,0001$, $\eta^{2} \mathrm{p}=0,417$. El análisis post-hoc indicó un aumento de la hostilidad en el grupo expuesto a Ruido Blanco (ver Tabla 1).

Tabla I. Medidas de EVEA

\begin{tabular}{|c|c|c|c|c|c|c|c|c|}
\hline & \multicolumn{2}{|c|}{ ANSIEDAD } & \multicolumn{2}{|c|}{ DEPRESIÓN } & \multicolumn{2}{|c|}{ ALEGRÍA } & \multicolumn{2}{|c|}{ HOSTILIDAD } \\
\hline & pre & post & pre & post & pre & post & pre & post \\
\hline RB & 3 & 4,1 & $\mathrm{I}, 7$ & 1,5 & 7,4 & 6,5 & 1 & $3,3 *$ \\
\hline ACT & 3,6 & 2,7 & $\mathrm{I}, 8$ & $\mathrm{I}, 3$ & 6,2 & 6,5 & 1,6 & 1,2 \\
\hline REL & 2,8 & $\mathrm{I}, 8^{*}$ & $\mathrm{I}, 8$ & 1,9 & 6,7 & 6,88 & $\mathrm{I}, 3$ & I \\
\hline
\end{tabular}

Media de EVEA pre y post tratamiento

* Se halló una diferencia significativa entre las medidas pre y post tratamiento 


\section{Recuerdo inmediato}

El ANOVA MR arrojó un efecto significativo del factor Condición $\mathrm{F}(2,29)=6,750, \mathrm{p}>0,05, \eta^{2} \mathrm{p}=0,310$; Palabra $\mathrm{F}(2,29)=29,016, \mathrm{p}<0,0001, \eta^{2} \mathrm{p}=0,492$; $\mathrm{y}$ de la interacción de Palabra x Condición F $(4,60)$ $=3,898, p=0,007, \eta^{2} p=0,206$. Los análisis post hoc en relación al factor Condición indicaron que el grupo Activante recordó mayor cantidad de palabras que los grupos Ruido Blanco y Relajante, quienes no difirieron entre sí. Los análisis posteriores en relación al tipo de palabra mostraron que las palabras negativas fueron mayormente recordadas que las positivas, y éstas a su vez que las neutras, en los tres grupos. Al analizar la interacción Palabra x Condición, se halló lo siguiente: los grupos no difirieron entre sí en relación a las palabras positivas y neutras; y el grupo Activante recordó mayor cantidad de palabras negativas que los otros dos grupos (quienes no difirieron entre sí). Los resultados se muestran en la Tabla 2.

Tabla 2. Puntajes ponderados

\begin{tabular}{lccc}
\hline & \multicolumn{3}{c}{ Recuerdo inmediato ponderado } \\
\cline { 2 - 4 } & Positivas & Negativas & Neutras \\
\hline RB & $0,3 \pm 0,2$ & $0,4 \pm 0,1$ & $0,2 \pm 0,1$ \\
\hline ACT & $0,4 \pm 0,2$ & $0,7 \pm 0,2$ & $0,3 \pm 0,2$ \\
\hline REL & $0,3 \pm 0,1$ & $0,3 \pm 0, I$ & $0,2 \pm 0,1$ \\
\hline
\end{tabular}

Medias y desvíos de puntajes ponderados de palabras negativas, positivas y neutras, ponderando los puntajes

\section{Discusión}

El objetivo del presente trabajo fue estudiar el efecto de la música activante y relajante sobre la memoria verbal emocional en adultos, y analizar el efecto de la congruencia del estado de ánimo sobre el tipo de material recordado. Los resultados indican que la música modula recuerdos, tanto emocionales como neutros, comprobando la primera hipótesis propuesta. Sin embargo, no se halló el efecto de congruencia esperado, ya que el grupo expuesto a música activante recordó mayor cantidad de palabras negativas y neutras, pero no de positivas.

Estudios previos indican que la activación emocional generada por la música incide en el recuerdo de material verbal, en la etapa de consolidación (Díaz Abrahan \& Justel, 2020). Algunos estudios sugieren que es la música activante la que modula los recuerdos (Justel et al., 2016; Rickard et al., 2012), concordando con nuestros hallazgos. Si bien la música relajante también mejoró los recuerdos de estímulos emocio- nales verbales (Moltrasio et al., 2020), no fue registrado en el presente estudio.

Cabe destacar que hubo mayor recuerdo de palabras negativas que positivas en las diversas condiciones. Esto no puede explicarse por los niveles de arousal despertados por éstas, ya que las palabras negativas y positivas activaron a los sujetos por igual. Es decir, consideramos que fue la valencia de los estímulos y no el arousal lo que determinó su mejor recuerdo. Este sesgo negativo del recuerdo había sido previamente reportado en adultos jóvenes (Ochsner, 2000). Además, se ha observado un aumento del procesamiento sensorial durante la codificación de material verbal negativo, relacionado con un mejor recuerdo posterior (Mickley \& Kensinger, 2008). Por otro lado, características individuales de personalidad, tales como alto neuroticismo, se asocia a mejor recuerdo de material negativo (Bradley, 1992; Rusting, 1999).

La música generó cambios en el estado de ánimo de los sujetos, aunque sólo se produjeron efectos en la reducción de la ansiedad con las condiciones musicales, y no hubo diferencias entre ellas. Sin embargo, esto se tradujo en un mejor recuerdo de palabras sólo para la condición Activante. El ruido blanco, que se suponía que actuaría como estímulo neutro, generó mayor hostilidad en los participantes (incluso comparado con los otros dos grupos). La reducción de la ansiedad utilizando música había sido previamente reportada (Bellier et al., 2020; Kühlmann et al., 2018), e incluso una mejora en la performance de profesionales expuestos a música relajante (Bellier et al., 2020). Es decir, la reducción de la ansiedad causada por la música pudo haber contribuido al mejor recuerdo del material. Cabe destacar, sin embargo, que la música relajante también produjo disminución de la ansiedad, pero no una mejora en el recuerdo.

Muchos estudios que utilizan música para inducir estados de ánimo le solicitan a los sujetos que se "sumerjan" en el estado de ánimo del estímulo musical, y esta diferencia podría haber causado que no en todos los casos se obtenga la inducción de estado de ánimo deseada (Clark, \& Teasdale, 1985; Martin, 1990; Oetken et al., 2017). Por otro lado, los estímulos musicales utilizados en el presente estudio difieren en arousal, y las medidas utilizadas para medir estado de ánimo, no evaluaron el nivel general de activación del sujeto como en otro estudio (Jallais, \& Gilet, 2010). Por lo tanto, pudo haberse subestimado la diferencia en el estado de ánimo provocada por los diferentes estímulos musicales. 
La hipótesis de congruencia no se vio apoyada en este estudio: los sujetos expuestos a música activante recordaron mayor cantidad de palabras negativas y totales que los otros dos, pero no se hallaron diferencias en las medidas de estado de ánimo pre y post-tratamiento en este grupo. Por otro lado, el estímulo de ruido blanco generó mayor hostilidad en los sujetos que las otras dos condiciones. Los sujetos expuestos a esta condición recordaron más palabras negativas que neutras. Estos resultados irían parcialmente en línea con la hipótesis de congruencia (Martin, 1990; Bower, 1981). Sin embargo, hubo un mejor recuerdo total de palabras negativas en todas las condiciones, con lo cual este efecto no fue específico para la condición de ruido blanco.

Los resultados contradictorios podrían explicarse, en principio, por el procedimiento utilizado. Los estudios que comprobaron la hipótesis de congruencia, en su mayoría, inducen el estado de ánimo antes de presentar el material a recordar (Tesoriero \& Rickard, 2012; Lewis et al., 2005). Se ha sugerido que la hipótesis de que la música modula los recuerdos debido a la activación emocional que produce, independientemente de la valencia de la misma, se ajusta mejor a los procedimientos que utilizan la música como tratamiento post-aprendizaje (Tesoriero \& Rickard, 2012). Además, algunos estudios utilizan mayores tiempos de exposición a la música para cambiar el estado anímico (Västfjäll, 2001). Los estados anímicos pueden ser el resultado de diversos eventos y, mientras una pieza musical breve puede activar respuestas emocionales intensas, probablemente un extracto musical debería ser más largo para producir un efecto en el estado de ánimo (Garrido et al., 2017).

Por otro lado, los adjetivos y palabras autorreferenciales muestran mayor congruencia que los sustantivos con la inducción de estado de ánimo utilizando música (Clark \& Teasdale, 1985; Knight et al., 2002), y las palabras empleadas en el presente estudio eran sustantivos. Como fue expuesto, algunas diferencias de personalidad podrían explicar la ausencia de congruencia. Otro factor asociado a la ausencia de congruencia es la dificultad de los sujetos para mantenerse o ser conscientes de sus propios estados anímicos (“mood repair") (Tesoriero \& Rickard, 2012). También se observó ausencia de congruencia en hombres, comparados con mujeres (Mayer et al., 1990), aunque nuestra muestra consistió mayormente de mujeres.

Dentro de las limitaciones halladas en el presente estudio se encuentra el hecho de que se haya utilizado solamente música con valencia positiva. Sumando una condición con música de valencia negativa y ac- tivante, podría explorarse si hay congruencia con el recuerdo de material negativo, y determinar si es el arousal del estímulo musical (independientemente de la valencia) lo que genera un mejor recuerdo. Por otro lado, el número de sujetos en cada grupo de nuestra muestra es pequeño.

En conclusión, nuestros hallazgos van en línea con estudios anteriores, que explican la modulación de los recuerdos en la etapa de consolidación, utilizando material emocionalmente activante. La música generó un mejor recuerdo de material verbal, tanto emocional como neutro. No se halló evidencia a favor de la hipótesis de congruencia. Es decir, hallamos evidencia a favor de la utilización de la música como moduladora de los recuerdos no congruentes con su valencia, destacando la importancia del arousal del estímulo musical. Estos resultados hallados en adultos jóvenes, permiten pensar el papel de la música como moduladora de la memoria. Futuras investigaciones podrían aplicar protocolos similares en poblaciones de músicos, a fin de explorar si la música modula de manera diferente el estado de ánimo y si esto influye en el tipo de material a recordar. Por otro lado, podría pensarse su aplicación experimental y eventualmente terapéutica, en patologías psiquiátricas y neurológicas que afecten los recuerdos emocionales, a fin de modular (aumentando o disminuyendo) los mismos.

Conflicto de intereses: las autoras declaran no tener conflictos de intereses. El presente trabajo se realizó en el marco de la beca doctoral UBACyT 20220170100038BA, enmarcada en el subsidio UBA $20020170100282 B A$.

\section{Referencias bibliográficas}

Beck, A., Steer, R., \& Brown, G. (2006). BDI-II-Inventario de Depresión de Beck. Paidós.

Bellier, A., Secheresse, T., Stoeckle, A., Dols, A. M., \& Chaffanjon, P. C. (2020). Impact of Background Music on Medical Student Anxiety and Performance During Anatomical Dissections: A Cluster Randomized Interventional Trial. Anatomical sciences education, 13(4), 427-435. https:// doi.org/10.1002/ase.1918

Bower, G. H. (1981). Mood and memory. The American psychologist, 36(2), 129-148. https://doi.org/10.1037//0003-066x.36.2.129

Bradley, M. M., Greenwald, M. K., Petry, M. C., \& Lang, P. J. (1992). Remembering pictures: Pleasure and arousal in memory. Journal of Experimental Psychology: Learning, Memory, and Cognition, 18(2), 379-390. https://doi.org/10.1037/0278-7393.18.2.379

Bradley, M. M., \& Lang, P. J. (1999). Affective norms for English words (ANEW): Instruction manual and affective ratings. Technical report $C-1$, the center for research in psychophysiology.

Bradley, M. M., \& Lang, P. J. (2000). Measuring emotion: Behavior, feeling, and physiology. In R. D. Lane \& L. Nadel (Eds.), Cognitive neuroscience of emotion (pp. 242-276). Oxford University Press.

Bruner, G.C. (1990). Music, mood, and marketing. Journal of marketing, 54(4), 94-104. doi:10.2307/1251762 
Butman, J., Arizaga, R., Harris, P., Drake, M., Baumann, D., De Pascale, A., et al. (2001). El "Mini Mental State Examination" en Español. Normas para Buenos Aires. Rev Neurol Arg, 26(1), 11-5.

Cahill, L., \& McGaugh, J. L. (1995). A novel demonstration of enhanced memory associated with emotional arousal. Consciousness and cognition, 4(4), 410-21. doi: 10.1006/ccog.1995.1048

Cahill, L., Haier, R. J., Fallon, J., Alkire, M. T., Tang, C., Keator, D., et al. (1996). Amygdala activity at encoding correlated with long-term, free recall of emotional information. Proceedings of the National Academy of Sciences, 93(15), 8016-21. doi: 10.1073/pnas.93.15.8016

Clark, D. M., \& Teasdale, J. D. (1985). Constraints on the effects of mood on memory. Journal of personality and social psychology, 48(6), 1595.

Castro, C. (2018, 29-31 de Agosto). Uso de la música como estrategia de afrontamiento para la regulación emocional en adultos jóvenes. XX Congreso Argentino de Neuropsiquiatría y Neurociencia Cognitiva, Buenos Aires, Argentina.

Chanda, M. L., \& Levitin, D. J. (2013). The neurochemistry of music. Trends in cognitive sciences, 17(4), 179-93.

Díaz Abrahan, V. M., \& Justel, N. (2020). Uso de la música para modular la memoria: Una revisión sistemática. Revista Iberoamericana De Psicología, 12(2), 39-50. https://doi.org/10.33881/2027-1786.rip.12204

Folstein, M. F., Folstein, S. E., \& McHugh, P. R. (1975). "Mini-mental state". A practical method for grading the cognitive state of patients for the clinician. Journal of psychiatric research, 12(3), 189-198. https://doi. org/10.1016/0022-3956(75)90026-6

Garrido, S., Bangert, D., \& Schubert, E. (2017). Mood induction studies : distinguishing between emotional response and long-term mood impacts of music. In SAGE Research Methods Cases. Part 2 (pp. 1-14). https://doi. org $/ 10.4135 / 9781473974760$

Jallais, C., \& Gilet, A-L. (2010). Inducing changes in arousal and valence: Comparison of two mood induction procedures. Behavior research methods, 42(1), 318-25. https://doi.org/10.3758/BRM.42.1.318

Judde, S., \& Rickard, N. (2010). The effect of post-learning presentation of music on long-term word-list retention. Neurobiology of learning and memory, 94(1), 13-20. https://doi.org/10.1016/j.nlm.2010.03.002

Justel, N., Psyrdellis, M., Ruetti, E. (2013). Modulación de la memoria emocional: una revisión de los principales factores que afectan los recuerdos. Suma psicológica, 20(2), 163-74. doi: 10.14349/sumapsi2013.1276

Justel, N., \& Rubinstein, W. Y. (2013). La exposición a la música favorece la consolidación de los recuerdos. Boletín de Psicología, (109),73-83.

Justel, N., O’Conor, J., Rubinstein, W. (2015). Modulación de la memoria emocional a través de la música en adultos mayores: Un estudio preliminar. Interdisciplinaria, 32(2), 247-59.

Justel, N., Diaz Abrahan, V., Castro, C., Rubinstein, W. (2016). Efecto de la música sobre la memoria emocional verbal. Anuario de Investigaciones, 21.

Kensinger, E.A., \& Corkin S. (2003). Memory enhancement for emotional words: Are emotional words more vividly remembered than neutral words? Memory \& cognition, 31(8), 1169-80. doi: 10.3758/bf03195800.

Knight, W. E., \& Rickard, N. S. (2001). Relaxing music prevents stress-induced increases in subjective anxiety, systolic blood pressure, and heart rate in healthy males and females. Journal of music therapy, 38(4), 254-272. https://doi.org/10.1093/jmt/38.4.254

Knight, B.G., Maines, M.L., \& Robinson, G.S. (2002). The effects of sad mood on memory in older adults: A test of the mood congruence effect. Psychology and aging. 17(4): 653.

Kreutz, G., Ott, U., Teichmann D., Osawa .P, Vaitl D. (2008). Using music to induce emotions: Influences of musical preference and absorption. Psychology of music, 36(1), 101-26. doi:10.1177/0305735607082623

Kühlmann, A., de Rooij, A., Kroese, L. F., van Dijk, M., Hunink, M., \& Jeekel, J. (2018). Meta-analysis evaluating music interventions for anxiety and pain in surgery. The British journal of surgery, 105(7), 773-783. https:// doi.org/10.1002/bjs.10853

Lewis, P. A., Critchley, H. D., Smith, A. P., \& Dolan, R. J. (2005). Brain mechanisms for mood congruent memory facilitation. NeuroImage, 25(4), 1214-1223. https://doi.org/10.1016/j.neuroimage.2004.11.053
Lundqvist, L.-O., Carlsson, F., Hilmersson, P., \& Juslin, P. N. (2009). Emotional responses to music: Experience, expression, and physiology. Psychology of Music, 37(1), 61-90. https://doi.org/10.1177/0305735607086048

Martin, M. (1990). On the induction of mood. Clinical Psychology Review, 10(6), 669-97.

Mayer, J. D., Gayle, M., Meehan, M. E., \& Haarman, A-K. (1990). Toward better specification of the mood-congruency effect in recall. Journal of Experimental Social Psychology, 26(6), 465-80. https://doi.org/10.1177/10298649020050S107

Mayer, J. D., McCormick, L. J., \& Strong, S. E. (1995). Mood-congruent memory and natural mood: New evidence. Personality and Social Psychology Bulletin, 21(7), 736-46.

Mercadal-Brotons, M., \& Augé, P.M. (2008). Manual de musicoterapia en geriatría y demencias: Monsa-Prayma.

Mickley, K. R., \& Kensinger, E. A. (2008). Emotional valence influences the neural correlates associated with remembering and knowing. Cognitive, affective \& behavioral neuroscience, 8(2), 143-152. https://doi.org/10.3758/ cabn.8.2.143

Moltrasio, J., Mora, M. J., Justel, N., Roncero, B. S., Politis, D. G, Rubinstein, W. (2020). Modulación de la memoria verbal con música activante y relajante. Cuadernos de Neuropsicología/Panamerican Journal of Neuropsychology, 14(3), 112-120.

Nasby, W. (1994). Moderators of mood-congruent encoding: Self-/ other-reference and affirmative/nonaffirmative judgement. Cognition \& Emotion, 8(3), 259-78. doi: 10.1080/02699939408408941

Ochsner, K. N. (2000). Are affective events richly recollected or simply familiar? The experience and process of recognizing feelings past. Journal of experimental psychology. General, 129(2), 242-261. https://doi. org/10.1037//0096-3445.129.2.242

Oetken, S., Pauly, K. D., Gur, R. C., Schneider, F., Habel, U., \& Pohl, A. (2017). Don't worry, be happy - Neural correlates of the influence of musically induced mood on self-evaluation. Neuropsychologia, 100, 26-34. https://doi.org/10.1016/j.neuropsychologia.2017.04.010

Redondo, J., Fraga, I., Padrón, I., \& Comesaña, M. (2007). The Spanish adaptation of ANEW (affective norms for English words). Behavior research methods, 39(3), 600-5. doi:10.3758/BF03193031

Rickard, N. S., Wong, W. W., \& Velik, L. (2012). Relaxing music counters heightened consolidation of emotional memory. Neurobiology of learning and memory, 97(2), 220-228. https://doi.org/10.1016/j.nlm.2011.12.005

Romero, C. J. R. (2018). Musicoterapia, habla y estado de ánimo. Areté, 18(1), 13-21.

Sanz Fernández, J. (2001). Un instrumento para evaluar la eficacia de los procedimientos de inducción de estado de ánimo: la "Escala de Valoración del Estado de Ánimo" (EVEA). Análisis y modificación de conducta, 27(111):71-110. http://www.uhu.es/publicaciones/ojs/index.php/amc/index Rusting, C. L. (1999). Interactive effects of personality and mood on emotion-congruent memory and judgment. Journal of Personality and Social Psychology, 77(5), 1073-1086. https://doi.org/10.1037/0022$\underline{3514.77 .5 .1073}$

Sheldon, S., Williams, K., Harrington, S., \& Otto, A. R. (2020). Emotional cue effects on accessing and elaborating upon autobiographical memories. Cognition, 198, 104217. https://doi.org/10.1016/j.cognition.2020.104217

Tesoriero, M., \& Rickard, N. S. (2012). Music-enhanced recall: An effect of mood congruence, emotion arousal or emotion function? Musicae Scientiae, 16(3), 340-56. doi:10.1177/1029864912459046

Västfäll, D. (2001). Emotion induction through music: A review of the musical mood induction procedure. Musicae Scientiae, 5(1_suppl): 173-211.

Vieillard, S., Peretz, I., Gosselin, N., Khalfa, S., Gagnon, L., \& Bouchard, B. (2008). Happy, sad, scary and peaceful musical excerpts for research on emotions. Cognition \& Emotion, 22(4), 720-52. doi:10.1080/02699930701503567 